\title{
A Common Genetic Influence on Human Intensity Ratings of Sugars and High-Potency Sweeteners
}

\author{
Liang-Dar Hwang, ${ }^{1,2}$ Gu Zhu, ${ }^{1}$ Paul A. S. Breslin, ${ }^{3,4}$ Danielle R. Reed, ${ }^{3}$ Nicholas G. Martin, ${ }^{1}$ and \\ Margaret J. Wright ${ }^{1}$ \\ ${ }^{1}$ OIMR Berghofer Medical Research Institute, Brisbane, Queensland, Australia \\ ${ }^{2}$ School of Medicine, University of Queensland, Brisbane, Queensland, Australia \\ ${ }^{3}$ Monell Chemical Senses Center, Philadelphia, Pennsylvania, USA \\ ${ }^{4}$ Department of Nutritional Sciences, School of Environmental and Biological Sciences, Rutgers University, New \\ Brunswick, New Jersey, USA
}

\begin{abstract}
The perception of sweetness varies among individuals but the sources of this variation are not fully understood. Here, in a sample of 1,901 adolescent and young adults (53.8\% female; $243 \mathrm{MZ}$ and $452 \mathrm{DZ}$ twin pairs, 511 unpaired individuals; mean age $16.2 \pm 2.8$, range 12-26 years), we studied the variation in the perception of sweetness intensity of two monosaccharides and two high-potency sweeteners: glucose, fructose, neohesperidine dihydrochalcone (NHDC), and aspartame. Perceived intensity for all sweeteners decreased with age (2-5\% per year) and increased with the history of otitis media (6-9\%). Males rated aspartame slightly stronger than females (7\%). We found similar heritabilities for sugars (glucose: $h^{2}=$ 0.31 , fructose: $h^{2}=0.34$ ) and high-potency sweeteners (NHDC: $h^{2}=0.31$, aspartame: $h^{2}=0.30$ ); all were in the modest range. Multivariate modeling showed that a common genetic factor accounted for $>75 \%$ of the genetic variance in the four sweeteners, suggesting that individual differences in perceived sweet intensity, which are partly due to genetic factors, may be attributed to a single set of genes. This study provided evidence of the shared genetic pathways between the perception of sugars and high-potency sweeteners.
\end{abstract}

Keywords: sweet taste, sweet intensity, perception, high-potency sweeteners, heritability, twins

The perception of sweet taste varies among individuals (Reed et al., 2006). Discovery of genetic variants in the sweet taste genes T1R3 and gustducin and their relationship with sucrose perception establish a role of inborn variation on sweet taste (Fushan et al., 2009; 2010; Mennella et al., 2012; 2014; 2015). Yet, a firm understanding of the moleculargenetic basis of human sweet perception remains undetermined.

New data from model organisms (i.e., mice) indicate there may be a second system to sense sweetness (Yee et al., 2011), in addition to T1R3 and gustducin. This second system is sensitive to caloric sugars but not high-potency sweeteners (Yee et al., 2011). In humans, genetic studies of sucrose perception, including perceived intensity, pleasantness, and preferred concentration, have suggested a heritability ranging from 0.14 to 0.55 (Bretz et al., 2006; Keskitalo et al., 2007a; 2007b; Knaapila et al., 2012; Mennella et al., 2005). However, there are no heritability estimates for high-potency sweeteners in humans, and the degree to which sugars and high-potency sweeteners share molecular mechanisms of sweet-taste transduction is not fully understood.

Here, in a large adolescent and young adult twin sample (695 complete twin pairs), we estimated the heritability of perceived intensity for four sweeteners. These included two commonly occurring natural saccharides, glucose and fructose (found in fruits and vegetables), and two high-potency sweeteners, aspartame and NHDC. Using multivariate genetic modeling, we examined the extent that any association between the four sweeteners was due to common environmental or genetic factors. Further, this modeling allowed us to investigate whether any of the genetic variance in the

RECEIVED 15 May 2015; ACCEPTED 20 May 2015.

ADDRESS FOR CORRESPONDENCE: Liang-Dar Hwang, Neuroimaging Genetics Group, QIMR Berghofer Medical Research Institute, Herston QLD 4006, Australia. E-mail: Daniel.Hwang@qimrberghofer.edu.au 


\section{TABLE 1}

Number of Families Before and After Data Screening

\begin{tabular}{lrr}
\hline Family type & Initial & After screening $^{\text {a }}$ \\
\hline MZ twin pairs & 234 & 189 \\
MZ twin pairs + sibling(s) & 82 & 54 \\
DZ twin pairs & 491 & 380 \\
DZ twin pairs + sibling(s) & 95 & 72 \\
Non-twin singletons/unpaired twins $^{b}$ & 150 & $320^{c}$
\end{tabular}

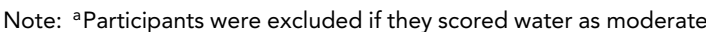
or higher taste ( $>20 \mathrm{~mm}$ on LMS), had large differences between presentation one and two and had overly high or low total average scores (Hansen et al., 2006). b Families with a twin pair and one or two siblings. 'The number of non-twin singletons/unpaired twins increases after cleaning as some twin pair families lose one twin during the screening procedure.

high-potency sweeteners could be attributed to a distinct set of genetic factors, separate to that for natural sugars.

\section{Materials and Methods}

\section{Participants}

Participants were adolescent and young adult twins and their singleton siblings from the Brisbane Adolescent Twin Study (Wright \& Martin, 2004) who have participated in previous studies of the genetics of melanoma risk factors (Aitken et al., 1994; Zhu et al., 1999), and cognition (Luciano et al., 2006; Wright et al., 2001). Taste data reported here were collected between August 2002 and July 2014. The sample comprised 1,175 females and 1,013 males (mean age $16.2 \pm 2.8$ years, range $12-26$ years) from 1,052 families, including $316 \mathrm{MZ}$ and $586 \mathrm{DZ}$ complete twin pairs, and 384 singletons (non-twin siblings and unpaired twins; Table 1). This includes all participants from a previous study of bitter perception (Hansen et al., 2006), with a $150 \%$ increase in sample size. Zygosity for $92 \%$ of the same-sex twins was determined from genotyping (Illumina 610K SNP array) and the remainder by self-report confirmed by study nurses. Participants (or their parents if under 18 years of age) gave written consent to participate in the study, which was approved by the Queensland Institute of Medical Research Human Research Ethics Committee.

\section{Taste Test}

As described previously, the taste test included both bitter and sweet stimuli (Hansen et al., 2006). Briefly, it included duplicate presentations of ten different solutions, of which five were bitter (propylthiouracil (PROP), sucrose octa-acetate (SOA), quinine $\mathrm{HCl}$, caffeine, and denatonium benzoate), four were sweet (described below), and one was neutral (i.e., water). The four sweet solutions included the two natural sugars, $0.60 \mathrm{M}$ glucose, $0.30 \mathrm{M}$ fructose, and two high-potency sweeteners, $8.0 \times 10^{-5} \mathrm{M}$ NHDC and $1.4 \times 10^{-3} \mathrm{M}$ aspartame. Each solution and the water control were presented twice (i.e., total of 20 solutions) in colorcoded $2 \mathrm{~mL}$ polypropylene microcentrifuge tubes with flip tops. The first 10 tubes contained one presentation of each compound plus the water control and the next 10 contained the same solutions in a different order, but the order of all 20 tubes was the same for all participants (SOA, water, caffeine, glucose, quinine $\mathrm{HCl}$, fructose, NHDC, PROP, aspartame, denatonium benzoate/ fructose, glucose, PROP, aspartame, quinine $\mathrm{HCl}$, NHDC, caffeine, water, SOA, denatonium benzoate). Participants were instructed to: (1) open the tube, swish the solution around in the mouth for five seconds, and spit out; (2) rate the perceived intensity of the solution; (3) rate the quality of the taste; (4) rinse the mouth out four times with tap water; and (5) repeat steps 1 to 4 for each tube. Perceived intensity was rated on a general labeled magnitude scale (LMS; Green et al., 1993) with labels of no sensation $(0 \mathrm{~mm})$, barely detectable $(2 \mathrm{~mm})$, weak $(7 \mathrm{~mm})$, moderate $(20 \mathrm{~mm})$, strong $(40 \mathrm{~mm})$, very strong $(61 \mathrm{~mm})$, and strongest imaginable $(114 \mathrm{~mm})$. This scale minimizes ceiling effects and provides a continuous measure that is desirable for quantitative analysis.

In addition, participants and/or a parent answered questions relating to a previous head injury and otitis media (middle ear infection), which are two common factors relating to the change in taste perception as they damage the signal transduction from the mouth to the brain (Bartoshuk et al., 1996; 2006; Schechter \& Henkin, 1974). History of head injury was coded as: (1) Never suffered from a head injury; (2) Yes, but not serious; (3) Yes, had either a concussion or loss of consciousness; (4) Yes, both concussion and loss of consciousness. History of otitis media was coded as: (0) Never suffered from middle ear infection and (1) Yes, had suffered from middle ear infection; $27 \%$ and $46 \%$ of participants had suffered from head injury and otitis media respectively. The taste test took 30-45 minutes, while total testing time for all components of the study was approximately two and a half hours.

Before March 2004, the taste test was self-administered as part of a mail and phone study. Test tubes were embedded in an inch-wide polyethylene sheet, rolled up into a padded post bag, and mailed to participants via regular post. The mail pack included both detailed written instructions and a summary sheet of the key points that participants could refer to while completing the test. Participants were instructed not to complete the taste test if suffering from a cold or flu until they had completely recovered, and not to eat or smoke, and drink only water for at least one hour before the test. Approximately, $40 \%$ of the data were collected via mail and the rest were collected during participants' visits at QIMR Berghofer Medical Research Institute.

\section{Data Screening}

Prior to analysis, the data were screened for outliers (Hansen et al., 2006). Briefly, participants were removed if they: (1) rated water stronger than moderate (mean score of first and second presentation $>20 \mathrm{~mm}) ;(2)$ had a low $(<200 \mathrm{~mm})$ or high $(>1800 \mathrm{~mm})$ total score across all 18 taste stimuli; or (3) if there was a large discrepancy $(>80 \mathrm{~mm})$ between 
ratings for the first and second presentations. This excluded $13 \%$ of the sample, with the final sample comprising 243 $\mathrm{MZ}$ and $452 \mathrm{DZ}$ twin pairs and their siblings, and 320 families of unpaired individuals (mean age of $16.2 \pm 2.8$ years; 1,023 females, 878 males; Table 1).

\section{Statistical Analyses}

Since intensity scores for all sweeteners were positively skewed, a square-root transformation was performed to approximate normal distributions. To establish regularity in sampling and measurement, and to check assumptions of the twin design, homogeneity of means and variances for birth order, and zygosity were tested using the statistical package Mx, which utilizes maximum likelihood (ML) estimation procedures (Neale et al., 2002). Outlying families were detected by using the percentage option in $\mathrm{Mx}$ that uses the Mahalanobis distance to identify families having excessive similarities or differences relative to other families in the sample and model expectation. As removal of outlying families (one to three families for fructose, NHDC, and aspartame) did not change any of the estimates, these families were included in all analyses. Covariates (sex, age, history of otitis media, and head injury) were tested for significance in fixed effects mean models (regressions and deviations from the mean). Models were assessed by comparing double the negative log-likelihood between nested models, as this difference is distributed asymptotically as a $\chi^{2}$, which is used to decide whether a model is a significant worse fit than its predecessor.

Variance components modeling partitions the variation of a trait into genetic and environmental sources by taking advantage of the differences in genetic relatedness between MZ (share all genes) and DZ (share half of genes) pairs. These known differences allow the estimation of additive genetic (A), non-additive genetic (D), common environment (C), and unique environment (E, includes experimental error) parameters in a variance components model. Because twins in this sample were reared together, the $C$ and $D$ parameters are negatively confounded and, as such, cannot be simultaneously estimated (Keller \& Coventry, 2005). However, if the MZ twin correlation is more than double the DZ twin correlation, it is indicative that non-additive genetic influences (including dominance and epistasis) are most important, whereas if the MZ twin correlation is less than double the DZ twin correlation, common environment is likely to be more important.

As there were two measurements for each compound, their differences were tested in univariate models and the phenotypic variation was further partitioned into a test unreliability $(\mathrm{U})$ component using a repeated measures model in which two presentations were treated as observations of one underlying score by constraining the two presentations to be equal. All variance components (A, C, and E) were then constrained to affect each presentation variable equally as both are imperfect measures of the true underlying phe- notype. Test unreliability (U) was then estimated from the variance not contributed by $\mathrm{A}, \mathrm{C}$, and $\mathrm{E}$.

As perceived taste intensities of the sweeteners were moderately correlated, we specified a multivariate model including all four compounds, utilizing the additional information gained from the cross-trait correlations to estimate common sources of variation between these traits. Three out of four $\mathrm{MZ}$ twin correlations were lower than double the DZ twin correlations, so a Cholesky decomposition of $\mathrm{A}, \mathrm{C}$, and $\mathrm{E}$ factors was used as a starting point, with covariates modeled as regressions or deviation effects on the mean. Alternative models of independent pathways and common pathways were also assessed to examine whether there were specific genetic components for all four variables. The best model fit was determined by Akaike's Information Criterion (AIC), which penalizes models for increasing complexity and can be used with non-nested models (Burnham \& Anderson, 2002).

\section{Results}

The mean perceived intensities of all four sweeteners were between moderate and strong (Table 2). There is a $5-15 \%$ difference between first and second presentations for each sweetener. Head injury had no significant effect on any phenotype while age, sex, and otitis media significantly $(p<.05)$ influenced some measures (Table 2). Between the ages of 12 and 26, perceived intensities of all four sweeteners decreased with age (2-5\% per year). For example, a 14 -year-old participant would rate the glucose solution 6.4 higher than a 24-year-old participant. Males rated aspartame 7\% more intense than females (27.6 vs. 25.7, $p=.015$ ). History of otitis media had a small but consistent positive effect on all sweeteners, with participants who had suffered from middle ear infections rating sweeteners as $6-9 \%$ more intense than those who had never had a middle ear infection. Phenotypic correlations among the four sweeteners (0.4-0.64) were similar to those between duplicate presentations of the same compound (0.48-0.58) (Table 3).

\section{Variance Components Modeling}

For all four sweeteners, the MZ twin correlations (0.27$0.33)$ were higher than the DZ twin correlations (0.120.22 ), though both correlations were low-modest (Table 2). Univariate ACE models showed no significant worsening of fit after removal of the shared environmental factor for each of the four sweeteners ( $\Delta$-2LL ranged from 0 to 1.55$)$. NHDC was further tested in an ADE model as the MZ correlation was more than double the DZ correlation. Removal of the dominant genetic factor from the ADE model did not result in a worse fit for $\operatorname{NHDC}(\Delta-2 \mathrm{LL}=0)$. As we found test unreliability (U) accounted for $40-50 \%$ of the variance for each sweetener (AEU model; Table 2), in all further modeling we used mean intensity, which is a more stable measure. 
TABLE 2

Sweet Intensity Characteristics of the Twin Sample

\begin{tabular}{|c|c|c|c|c|}
\hline & Glucose & Fructose & $\mathrm{NHDC}^{\mathrm{d}}$ & Aspartame \\
\hline \multicolumn{5}{|l|}{ Descriptive statistics } \\
\hline Pre1 $(N=1,876-1,888)^{a}$ & $33.6 \pm 18.2$ & $32.6 \pm 20$ & $33.2 \pm 20.4$ & $27.3 \pm 18.6$ \\
\hline Pre2 $(N=1,868-1,873)^{a}$ & $29.2 \pm 18.5$ & $30.9 \pm 19.2$ & $35.8 \pm 21.7$ & $25.8 \pm 18$ \\
\hline Mean $(N=1,882-1,890)^{a}$ & $31.4 \pm 16$ & $31.8 \pm 17.7$ & $34.5 \pm 18.9$ & $26.6 \pm 16.4$ \\
\hline \multicolumn{5}{|l|}{ Covariate effects } \\
\hline $\operatorname{Sex}(95 \% \mathrm{Cl})$ & $0.01(-0.12,0.13)$ & $0.08(-0.06,0.22)$ & $0.05(-0.09,0.20)$ & $0.18(0.05,0.32)$ \\
\hline Age $(95 \% \mathrm{Cl})$ & $-0.06(-0.08,-0.03)$ & $-0.11(-0.13,-0.08)$ & $-0.11(-0.14,-0.08)$ & $-0.08(-0.11,-0.06)$ \\
\hline Otitis media $(95 \% \mathrm{Cl})$ & $0.19(0.07,0.31)$ & $0.19(0.06,0.33)$ & $0.22(0.09,0.36)$ & $0.15(0.02,0.29)$ \\
\hline \multicolumn{5}{|l|}{ Twin correlations } \\
\hline$r_{M Z}(95 \% C l)(238-240 \text { pairs) })^{a, b}$ & $0.31(0.19,0.42)$ & $0.33(0.21,0.44)$ & $0.32(0.2,0.43)$ & $0.27(0.15,0.39)$ \\
\hline$r_{D Z}(95 \% C l)(446-449 \text { pairs) })^{a, b}$ & $0.22(0.12,0.3)$ & $0.19(0.09,0.28)$ & $0.12(0.03,0.21)$ & $0.17(0.08,0.26)$ \\
\hline \multicolumn{5}{|l|}{ Univariate AEU modeling } \\
\hline $\mathrm{A}(95 \% \mathrm{Cls})^{\mathrm{c}}$ & $25 \%(18,32)$ & $26 \%(19,33)$ & $25 \%(17,32)$ & $23 \%(15,30)$ \\
\hline $\mathrm{E}(95 \% \mathrm{Cls})^{\mathrm{c}}$ & $25 \%(18,33)$ & $34 \%(26,41)$ & $32 \%(25,40)$ & $35 \%(27,43)$ \\
\hline $\mathrm{U}(95 \% \mathrm{Cls})^{\mathrm{c}}$ & $50 \%(46,53)$ & $40 \%(37,43)$ & $43 \%(40,46)$ & $42 \%(39,46)$ \\
\hline
\end{tabular}

Note: Means, standard deviations, and sample sizes for the perceived intensity (millimeter on a labeled magnitude scale) of sweeteners from presentation 1 and 2 and their means. Estimates of phenotypic variation accounted by unreliability (U). Age, sex, and otitis media covariate effect estimates, and twin correlations. The regression and deviations for covariate effects were applied to the means of the square-root transformed variables. Significant covariate effects are shown in bold. ${ }^{a}$ The number of participants $(N)$ changes between presentations, as not all participants completed the entire test. ${ }^{b}$ Twin correlations calculated using means. ' Estimates of $A$ (additive genetic component), $E$ (non-shared environmental component), and $U$ (unreliability component) add up to $100 \%$ variance for each sweetener. ${ }^{d}$ Neohesperidine dihydrochalcone.

TABLE 3

Phenotypic Correlation Coefficients Among the Perceived Intensity of Four Sweeteners

\begin{tabular}{|c|c|c|c|c|c|c|c|c|c|}
\hline & & \multicolumn{2}{|c|}{ Glucose } & \multicolumn{2}{|c|}{ Fructose } & \multicolumn{2}{|c|}{ NHDC ${ }^{a}$} & \multicolumn{2}{|c|}{ Aspartame } \\
\hline & & 1 & 2 & 1 & 2 & 1 & 2 & 1 & 2 \\
\hline \multirow[t]{2}{*}{ Glucose } & 1 & - & & & & & & & \\
\hline & 2 & 0.48 & - & & & & & & \\
\hline \multirow[t]{2}{*}{ Fructose } & 1 & 0.62 & 0.49 & - & & & & & \\
\hline & 2 & 0.51 & 0.63 & 0.58 & - & & & & \\
\hline \multirow{2}{*}{$\mathrm{NHDC}^{\mathrm{a}}$} & 1 & 0.51 & 0.50 & 0.64 & 0.54 & - & & & \\
\hline & 2 & 0.43 & 0.54 & 0.48 & 0.53 & 0.56 & - & & \\
\hline \multirow[t]{2}{*}{ Aspartame } & 1 & 0.48 & 0.52 & 0.55 & 0.54 & 0.54 & 0.47 & - & \\
\hline & 2 & 0.40 & 0.53 & 0.47 & 0.49 & 0.43 & 0.48 & 0.56 & - \\
\hline
\end{tabular}

Note: Two presentations for each sweetener. Coefficients between duplicate presentations of the same compounds shown in

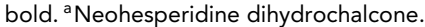

TABLE 4

Model Fit of the Multivariate Models for Perceived Intensity of Sweeteners

\begin{tabular}{|c|c|c|c|c|c|c|}
\hline Model & & $d f$ & $-2 \mathrm{LL}$ & $\Delta d f$ & $\Delta-2 \mathrm{LL}$ & $\mathrm{AlC}$ \\
\hline \multirow[t]{4}{*}{ Cholesky } & ACE & 7,495 & $16,572.79$ & - & - & $1,582.792$ \\
\hline & $A E^{a}$ & 7,505 & $16,576.29$ & 10 & 3.50 & $1,566.288$ \\
\hline & $\mathrm{CE}^{\mathrm{a}}$ & 7,505 & $16,583.39$ & 10 & 10.60 & $1,573.389$ \\
\hline & $\mathrm{E}^{\mathrm{a}}$ & 7,515 & $16,661.22$ & 20 & 88.43 & $1,631.222$ \\
\hline Common pathway & $\mathrm{AE}$ & 7,512 & $16,583.36$ & & & $1,559.356$ \\
\hline Independent pathway & $A E$ & 7,509 & $16,582.10$ & & & $1,564.096$ \\
\hline
\end{tabular}

Note: Abbreviations: degrees of freedom (df); -2 times the log-likelihood (-2LL); Akaike's information criterion (AIC). Best model shown

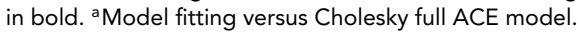

Model fit of the multivariate models, which included mean intensity ratings for each of the four sweeteners, are shown in Table 4. Dropping the shared environmental factors from the full ACE, Cholesky did not worsen the model fit $(\Delta-2 \mathrm{LL}=3.50, \Delta d f=10, \mathrm{AIC}=1566.288)$ and revealed one common genetic factor and a specific genetic factor for fructose, NHDC, and aspartame. While the CE model (dropping genetic factors) also provided a better fit than the full (ACE) Cholesky, the AE model was a better fit according to the AIC. A common pathway model (AIC = 1559.356), shown in Figure 1, provided the best fit among the models tested (Table 4). From the independent pathway model, heritabilities were estimated to be $0.31,0.34,0.31$, and 0.30 for glucose, fructose, NHDC, and aspartame, respectively. A common genetic factor explained the majority of the genetic variance for glucose $(88 \%)$, fructose $(87 \%)$, NHDC (79\%), and aspartame (76\%) ratings, with specific factors accounting for only a small amount of the genetic 


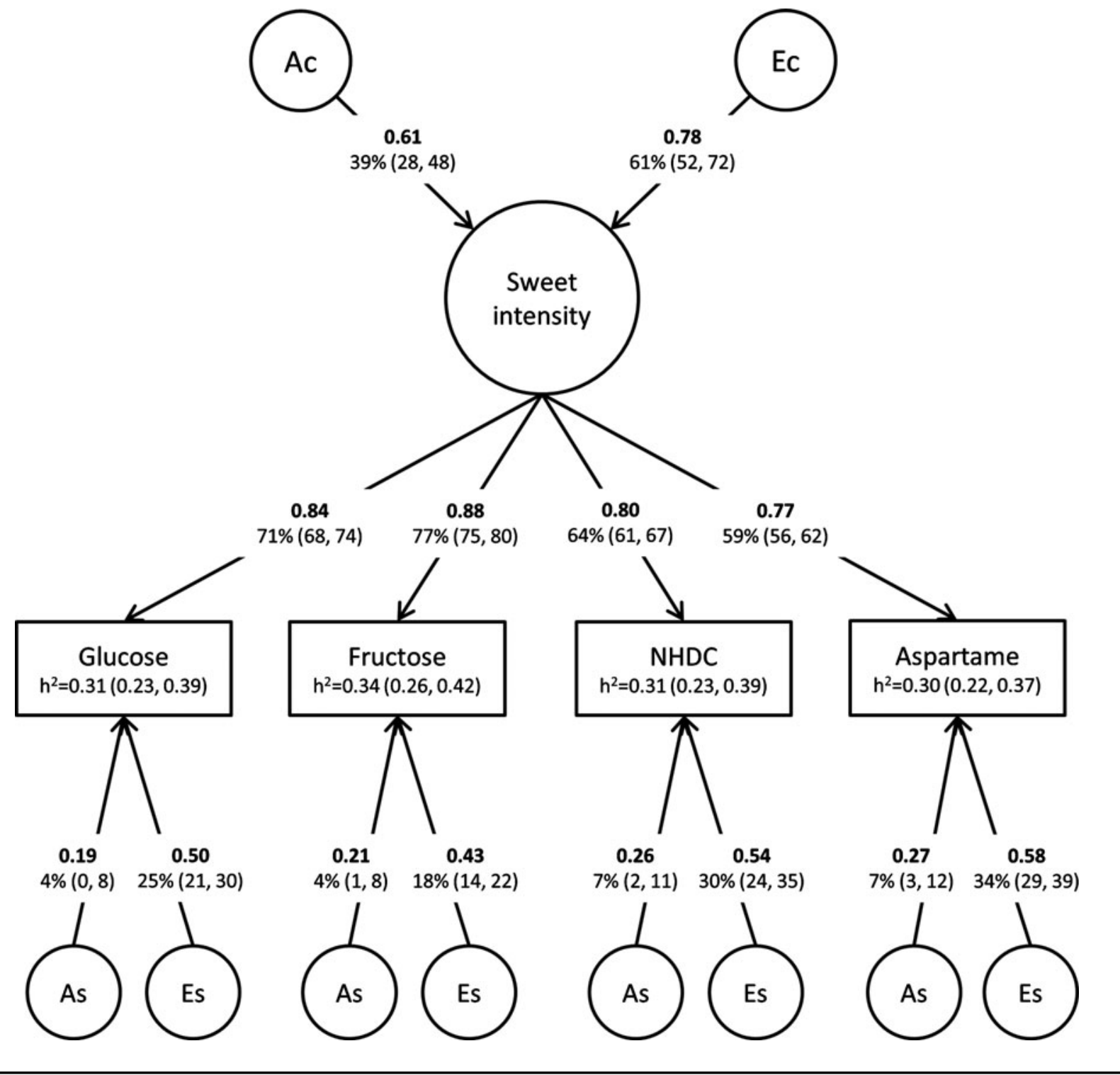

\section{FIGURE 1}

Common pathway model for perceived intensity of four sweeteners (standardized path coefficients and percentage of variance with $95 \% \mathrm{Cls})$. Ac and Ec are common additive genetic and environmental effects on the intensity measurement of four sweeteners. As and Es are specific genetic and environmental effects for each sweetener. Heritability estimates $\left(h^{2}\right)$ for each sweetener are sums of loadings from the Ac and As; for example, $h^{2}$ for glucose is $0.39 * 0.71+0.04$. Adding loadings from Ac, Ec, As, and Es gives a total variance of $100 \%$ for each sweetener.

variance $(12 \%$ to $24 \%)$. Genetic correlations ranged between 0.78 and 0.89 with the lowest between fructose and aspartame, and the highest between glucose and fructose. Environmental factors accounted for $66 \%$ to $70 \%$ of the variance.

\section{Discussion}

This is the first study to estimate the heritability and genetic covariance for perceived intensity of four structurally diverse sweet compounds: glucose, fructose, NHDC, and aspartame. Modest heritabilities were estimated at $0.31,0.34$, 0.31 , and 0.30 for glucose, fructose, NHDC, and aspartame, respectively. These estimates are similar to that previously reported for the sucrose intensity (Keskitalo et al., 2007b). Our finding that a common genetic factor accounts for most of the genetic variance suggests that a single set of genes influences the perceived intensity of all the sweeteners. This factor explained $23-30 \%$ of the phenotypic (total) variance, whereas specific additive genetic components accounted for $4-7 \%$ of the variance. Although there is no evidence for a specific genetic factor influencing the perception of caloric sugars, the common genetic factor could include parallel pathways that may account for a different amount of the genetic variance in the perception of sugars compared with high-potency sweeteners. 
We found little evidence for a shared environmental factor for sweet perception, which is also consistent with prior work examining sucrose intensity (Keskitalo et al., 2007b). Although there is a belief that diets high in sugar can change sweet preference, and sweet foods are often withheld from children early in life to modify this, our findings, and those of Keskitalo et al. (2007b) suggest that common environmental influences on sweet intensity ratings are very small. Similar data from animal models show that mice and rats, either exposed or not exposed to sugar early in life, do not differ as adults in sweet-related behavior (Bernstein et al., 1986; Wurtman \& Wurtman, 1979).

Univariate AEU modeling showed that test unreliability accounted for nearly $50 \%$ of the trait variation. This places an upper ceiling on the heritability estimate by removing variance that can be in part explained by genotype and thus results in an underestimation of the heritability. Unreliability of sweet intensity is commonly found among researchers because sweet responses are subject to influences of circulating insulin, glucagon, leptin, and cannabinoids, which vary from meal to meal, time of day, and from day to day (Nakamura et al., 2008; Yoshida et al., 2010). However, as all sweeteners were tested within a small time frame in this study, the unreliability was more likely to be measurement specific. Therefore, a more stable measure of mean intensity was used and the heritability estimates were similar to those previously reported (Keskitalo et al., 2007b).

We found that the effect of age on sweet perception was similar to that for bitter (Hansen et al., 2006), suggesting that as adolescents move from childhood to adulthood, the intensity of both sweet and bitter tastes decreases. Despite the cross-sectional design of this study, the age effect suggests that the taste systems are changing from child-like to adult-like; the results of this study suggest that during this period, the perception of intensity from sweet compounds decreases (Mennella et al., 2014). Likewise, longitudinal studies of sweet preference in both humans and mice revealed a negative age effect (Bertino \& Wehmer, 1981; Desor \& Beauchamp, 1987).

Similarly, history of otitis media positively affected the perceived intensity of all sweet measures. It has been proposed previously that the damage of otitis media infection to the chorda tympani, a taste nerve from the tongue to the brain that passes behind the tympanum, results in an increase in the number of taste buds per fungiform papilla (Bartoshuk et al., 1996). More taste buds correspond to more taste receptor cells, and consequently may influence sweetness perception. As a positive effect of otitis media was also observed for bitter compounds (Hansen et al., 2006), it may be that otitis media infection causes a global increase in the intensity of both sweet and bitter tastes.

In contrast to the global effect of age and otitis media, we found a sex effect only for aspartame, with males rating aspartame slightly more intense than females. Previous studies of smaller sample size reported no sex influences on the perceived intensity of aspartame, but only on other perception measures such as sweetness and pleasantness (Thai et al., 2011; Tordoff \& Alleva, 1990); this suggests the complexity in human perception of sweetness.

In conclusion, this study has examined the perceived intensity of four structurally diverse sweet compounds: two naturally occurring saccharides (glucose and fructose) and two high-potency sweeteners commonly used as additives to human foods (NHDC and aspartame). We have established that a moderate amount of the variation in the perceived intensity of sweeteners is due to genetic factors, and that there appears to be a single set of genes responsible for most of the variation in perception of these four compounds. This suggests that genetic factor scores for sweet intensity may be optimal for genome-wide association analyses.

\section{Acknowledgments}

We thank Kirsten J Mascioli, Christopher Tharp, Fujiko Duke, Deborah Lee, and Corrine Mansfield from the Monell Chemical Senses Center for manufacturing the taste tests; and from the QIMR Berghofer, Marlene Grace, Ann Eldridge, Natalie Garden, Kerrie McAloney for project coordination, data collection, and data entry, and David Smyth and Anthony Conciatore for computer support. In particular, thanks go to twins and their families for their participation. This work was supported by the National Institute of Health, grants DC02995 to PASB and DC004698 to DRR and the Australian NHMRC grants 241944 and 1031119.

\section{References}

Aitken, J. F., Green, A., Eldridge, A., Green, L., Pfitzner, J., Battistutta, D., ... Martin, N. G. (1994). Comparability of naevus counts between and within examiners, and comparison with computer image analysis. British Journal of Cancer, 69, 487-491.

Bartoshuk, L. M., Duffy, V. B., Hayes, J. E., Moskowitz, H. R., \& Snyder, D. J. (2006). Psychophysics of sweet and fat perception in obesity: Problems, solutions, and new perspectives. Philosophical Transactions of the Royal Society of London. Series B: Biological Sciences, 361, 1137-1148.

Bartoshuk, L. M., Duffy, V. B., Reed, D., \& Williams, A. (1996). Supertasting, earaches, and head injury: Genetics and pathology alter our taste. Neuroscience Biobehavioral Research, 20, 79-87.

Bernstein, I. L., Fenner, D. P., \& Diaz, J. (1986). Influence of taste stimulation during the suckling period on adult taste preference in rats. Physiology \& Behavior, 36, 913-919.

Bertino, M., \& Wehmer, F. (1981). Dietary influences on the development of sucrose acceptability in rats. Developmental Psychobiology, 14, 19-28.

Bretz, W. A., Corby, P. M., Melo, M. R., Coelho, M. Q., Costa, S. M., Robinson, M., ... Hart, T. C. (2006). Heritability estimates for dental caries and sucrose sweetness preference. Archives of Oral Biology, 51, 1156-1160. 
Burnham, K. P., \& Anderson, D. R. (2002). Model selection and multimodel inference: A practical information-theoretic approach. New York: Springer Science \& Business Media.

Desor, J. A., \& Beauchamp, G. K. (1987). Longitudinal changes in sweet preferences in humans. Physiology \& Behavior, 39, 639-641.

Fushan, A. A., Simons, C. T., Slack, J. P., \& Drayna, D. (2010). Association between common variation in genes encoding sweet taste signaling components and human sucrose perception. Chemical Senses, 35, 579-592.

Fushan, A. A., Simons, C. T., Slack, J. P., Manichaikul, A., \& Drayna, D. (2009). Allelic polymorphism within the TAS1R3 promoter is associated with human taste sensitivity to sucrose. Current Biology, 19, 1288-1293.

Green, B. G., Shaffer, G. S., \& Gilmore, M. (1993). Derivation and evaluation of a semantic scale of oral sensation magnitude with apparent ratio properties. Chemical Senses, 18, 683-702.

Hansen, J. L., Reed, D. R., Wright, M. J., Martin, N. G., \& Breslin, P. A. (2006). Heritability and genetic covariation of sensitivity to $\mathrm{PROP}, \mathrm{SOA}$, quinine $\mathrm{HCl}$, and caffeine. Chemical Senses, 31, 403-413.

Keller, M. C., \& Coventry, W. L. (2005). Quantifying and addressing parameter indeterminacy in the classical twin design. Twin Research and Human Genetics, 8, 201-213.

Keskitalo, K., Knaapila, A., Kallela, M., Palotie, A., Wessman, M., Sammalisto, S., .. . Perola, M. (2007a). Sweet taste preferences are partly genetically determined: Identification of a trait locus on chromosome 16. American Journal of Clinical Nutrition, 86, 55-63.

Keskitalo, K., Tuorila, H., Spector, T. D., Cherkas, L. F., Knaapila, A., Silventoinen, K. ... Perola, M. (2007b). Same genetic components underlie different measures of sweet taste preference. American Journal of Clinical Nutrition, 86, 1663-1669.

Knaapila, A., Hwang, L. D., Lysenko, A., Duke, F. F., Fesi, B., Khoshnevisan, A., ... Reed, D. R. (2012). Genetic analysis of chemosensory traits in human twins. Chemical Senses, 37, 869-881.

Luciano, M., Wright, M. J., Duffy, D. L., Wainwright, M. A., Zhu, G., Evans, D. M., ... Martin, N. G. (2006). Genomewide scan of IQ finds significant linkage to a quantitative trait locus on 2q. Behavior Genetics, 36, 45-55.

Mennella, J. A., Finkbeiner, S., Lipchock, S. V., Hwang, L. D., \& Reed, D. R. (2014). Preferences for salty and sweet tastes are elevated and related to each other during childhood. PLoS One, 9, e92201.

Mennella, J. A., Finkbeiner, S., \& Reed, D. R. (2012). The proof is in the pudding: Children prefer lower fat but higher sugar than do mothers. International Journal of Obesity, 36, 12851291.

Mennella, J. A., Pepino, M. Y., \& Reed, D. R. (2005). Genetic and environmental determinants of bitter perception and sweet preferences. Pediatrics, 115, e216-e222.
Mennella, J. A., Reed, D. R., Mathew, P. S., Roberts, K. M., \& Mansfield, C. J. (2015). 'A spoonful of sugar helps the medicine go down': Bitter masking by sucrose among children and adults. Chem Senses, 40, 17-25.

Nakamura, Y., Sanematsu, K., Ohta, R., Shirosaki, S., Koyano, K., Nonaka, K., ... Ninomiya, Y. (2008). Diurnal variation of human sweet taste recognition thresholds is correlated with plasma leptin levels. Diabetes, 57, 26612665.

Neale, M. C., Boker, S. M., Xie, G., \& Maes, H. H. (2002). Mx: Statistical modeling (6th ed.), Richmond, VA: Department of Psychiatry, Virginia Commonwealth University.

Reed, D. R., Tanaka, T., \& McDaniel, A. H. (2006). Diverse tastes: Genetics of sweet and bitter perception. Physiology \& behavior, 88, 215-226.

Schechter, P. J., \& Henkin, R. I. (1974). Abnormalities of taste and smell after head trauma. Journal of Neurology, Neurosurgery \& Psychiatry, 37, 802-810.

Thai, P.-K., Tan, E.-C., Tan, W.-L., Tey, T.-H., Kaur, H., ... Say, Y.-H. (2011). Sweetness intensity perception and pleasantness ratings of sucrose, aspartame solutions, and cola among multi-ethnic Malaysian subjects. Food Quality and Preference, 22, 281-289.

Tordoff, M. G., \& Alleva, A. M. (1990). Oral stimulation with aspartame increases hunger. Physiology \& Behavior, 47, $555-559$.

Wright, M. J., De Geus, E., Ando, J., Luciano, M., Posthuma, D., Ona, Y., ... Boomsma, D. (2001). Genetics of cognition: Outline of a collaborative twin study. Twin Research, 4, 4856.

Wright, M. J., \& Martin, N. G. (2004). Brisbane adolescent twin study: Outline of study methods and research projects. Australian Journal of Psychology, 56, 65-78.

Wurtman, J. J., \& Wurtman, R. J. (1979). Sucrose consumption early in life fails to modify the appetite of adult rats for sweet foods. Science, 295, 321-322.

Yee, K. K., Sukumaran, S. K., Kotha, R., Gilbertson, T. A., \& Margolskee, R. F. (2011). Glucose transporters and ATPgated $\mathrm{K}+(\mathrm{KATP})$ metabolic sensors are present in type 1 taste receptor 3 (T1r3)-expressing taste cells. Proceedings of the National Academy of Sciences of the United States of America, 108, 5431-5436.

Yoshida, R., Ohkuri, T., Jyotaki, M., Yasuo, T., Horio, N., Yasumatsu, K., .. . Ninomiya, Y. (2010). Endocannabinoids selectively enhance sweet taste. Proceedings of the National Academy of Sciences of the United States of America, 107, 935-939.

Zhu, G., Duffy, D. L., Eldridge, A., Grace, M., Mayne, C., O’Gorman, L., ... Martin, N. G. (1999). A major quantitative-trait locus for mole density is linked to the familial melanoma gene CDKN2A: A maximum-likelihood combined linkage and association analysis in twins and their sibs. American Journal of Human Genetics, 65, 483-492. 